\title{
Untersuchungen über die körperlichen Störungen bei der Schizophrenie.
}

\author{
Von \\ Dr. 0. Wuth (München). \\ (Eingegangen am 19. Mai 1922.*)
}

Meine Damen und Herren! Die Erwägungen, welche zur Anstellung der Untersuchungen, über die ich Ihnen nachher berichten will, geführt haben, waren in der Hauptsache differentialdiagnostische, entstanden aus der Erkenntnis, daß es uns heute in vielen Fällen mit den jetzigen Hilfsmitteln nicht gelingt, eine Diagnose zu sichern. Der Wunsch nun, etwa in Ähnlichkeit mit der Wassermann schen Reaktion, ein brauchbares körperliches Kriterium zu finden, veranlaßte mich serologische, morphologische und chemische Blutuntersuchungen bei den 4 Hauptgruppen von Psychosen, nämlich der Dementia praecox, dem manisch-depressiven Irresein, der Paralyse und der Epilepsie anzustellen. Die Auswahl der Untersuchungsmethoden mußte sich zum Teil nach den bereits vorliegenden Arbeiten richten. Es wurden infolgedessen auch Methoden in den Arbeitsplan aufgenommen, mit denen unserer Überzeugung nach nicht viel anzufangen war. Der Kürze halber muß ich mir es versagen, auf Wert und Unwert der einzelnen Reaktionen, sowie auf die Methodik hier einzugehen. Die Untersuchungen erstrecken sich auf 160 Geisteskranke und zwar je 40 Melancholische, Schizophrene, Epileptiker und Paralytiker.

Über die Resultate dieser Untersuchungen möchte ich Ihnen, soweit sie auf die Dementia praecox Bezug haben, nunmehr in tunlichster Kürze einige Mitteilungen machen und zugleich die möglichen Schlußfolgerungen auf das Wesen dieser Krankheit beleuchten.

Wie sie wissen, beobachten wir bei der Dementia praecox häufig eine Reihe von körperlichen Störungen. Abgesehen von den wichtigsten Wachstumsanomalien, wie Hochwuchs, Infantilismus seien hier die Schwankungen des Körpergewichts, der Motilitätsstörungen, die Temperaturschwankungen, sowie die Störungen auf dem Gebiete des vegetativen Nervensystems erwähnt. Eben diese Störungen in Gemeinschaft mit dem Verblödungsprozeß sind es, welche ein weiteres Eindringen in die Somatologie der Dementia praecox rechtfertigen. Ohne auf Einzelheiten der bisherigen Forschung einzugehen, möchte

*) Der Vortrag ist entnommen den inzwischen im Druck erschienenen „Untersuchungen über die körperlichen Störungen bei Geisteskranken“. Verlag J. Springer, Berlin. 1922. 
ich die hauptsächlichsten über das Wesen der Dementia praecox aufgestellten Theorien kurz darlegen, um sodann an Hand meiner Untersuchungsergebnisse dazu Stellung zu nehmen. Die namentlich von französischen und englischen Autoren vertretene Hypothese von einer infektiös. toxischen Störung sei hier nur aus historischem Interesse erwähnt. Die meisten späteren Erklärungstheorien suchen für die Krankheitserscheinungen Toxine verantwortlich zu machen, deren Vorhandensein man aus beobachteten Schwankungen der Zahlen für die weißen Blutkörperchen, die eosinophilen Zellen, den antitryptischen Serumtiter und die Gerinnungszeit des Blutes schloß. Für die Entstehung solcher hypothetischen Toxine suchte man nun verschiedene Erklärungen. Einige Autoren nahmen eine Ausscheidungsstörung der Nieren an und stellten geradezu die Katatonie als Folge einer Retention von giftigen Stoffen dar. Ebenfalls auf der Annahme einer Retention giftiger Eiweißabkömmlinge in Verbindung mit pathologisch gesteigertem Zerfall im Sinne der Anaphylaxie basiert die Hypothese einer Eiweißzerfalls. toxikose, als deren Kennzeichen man Steigerung des antitryptischen Serumtiters und der Harntoxizität ansah. Die meisten Anhänger hat jedoch die Theorie der endokrinen Genese gefunden und man führt als Stütze dieser Hypothese die Zusammenhänge zwischen Schizophrenie und den Äußerungen des Geschlechtslebens an, sowie ferner Veränderungen des Blutes und des Tonus des vegetativen Nervensystems und schließlich die Resultate der Abderhalden schen Reaktion.

Nun zu meinen Untersuchungsergebnissen, die Sie in den folgenden Tafeln aufgezeichnet finden. Einmalige Untersuchungen des Serumeiweißes und damit auch des Wassergehaltes des Serums lassen, wie Tafeln 1-4 zeigen, keine Unterschiede zwischen der Schizophrenie und den anderen Psychosen erkennen. Einmalige Serumeiweißuntersuchungen sind überhaupt bei den fortgesetzten Schwankungen für unsere Zwecke fast wertlos. Dagegen ermöglichten fortlaufende Serum. eiweißuntersuchungen in einigen Fällen den Nachweis zu führen, daß es sich bei Gewichtsstürzen häufig um Wasserverluste handelt. Hierfür gebe ich zwei Beispiele:

Kurve 1 beginnt mit dem Abklingen des katatonischen Erregungszustandes, Kurve 2 stellt Gewichts- und Serum-Eiweißkurve einer völlig ruhigen Dementia - praecox-Kranken dar. Die Gegenläufigkeit der Kurven zeigt, daß es sich um Wasserverluste seitens des Organismus handelt.

Tafeln 5-8 zeigen die Eiweißquotienten, welche lediglich bei der Paralyse eine gewisse Erhöhung erkennen lassen. Die von Krause für die Dementia praecox behauptete Globulinvermehrung können wir somit nicht bestätigen, 
Kurve 1,

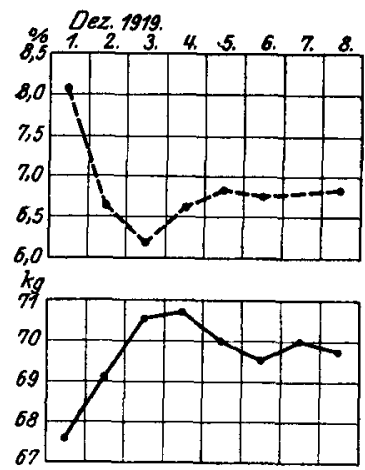

Kurve 2.

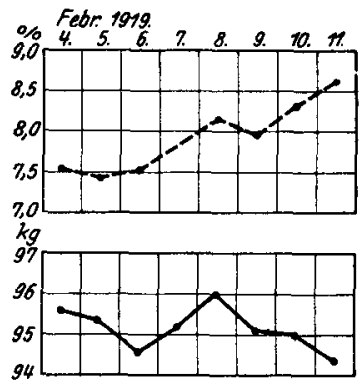

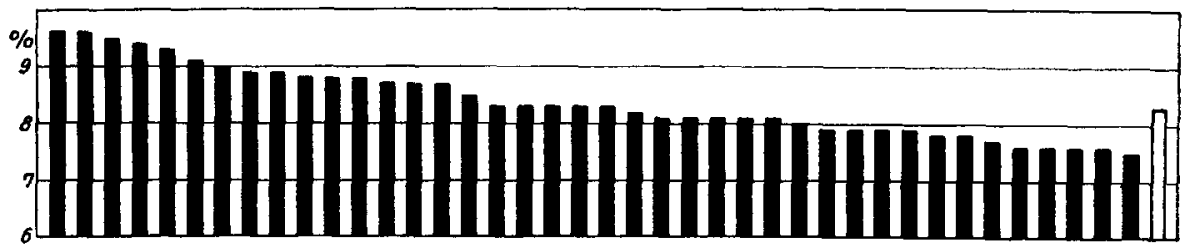

Abb. 1. Serumeiweiß : Melancholie.

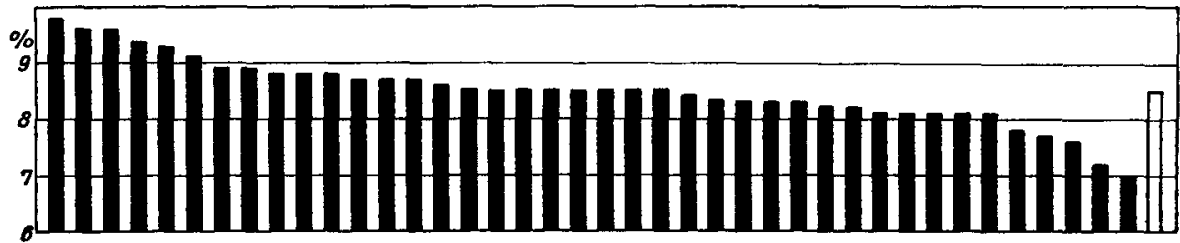

Abb. 2. Serumeiweib: Dementia praecox.

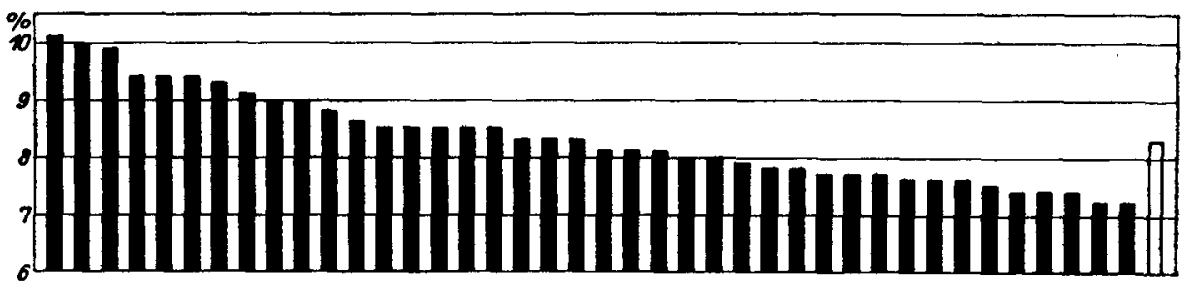

Abb. 3. SerumeivelB: Epilepsle.

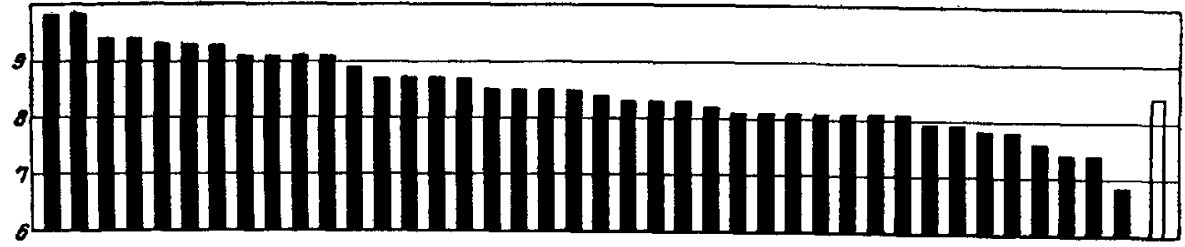

Abb. 4. BerumeiweíB : Paralyse. 


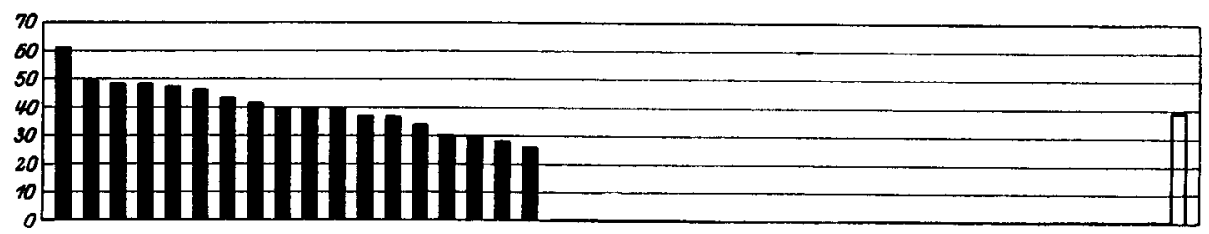

Abb. 5. Verhältnis des Globulins zum Gesamteiweiß in \%. Melancholie.

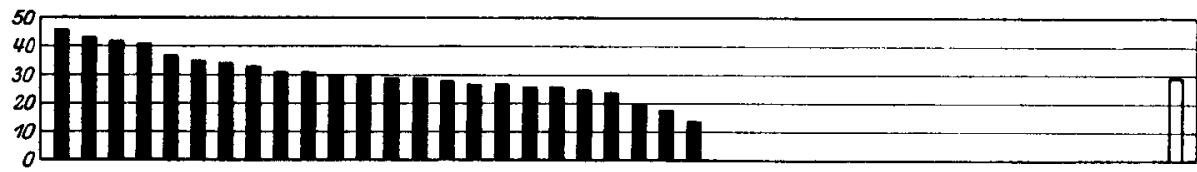

Abb. 6. Verhältnis des Globulins zum Gesamteiweiß in \%. Dementia praecox.

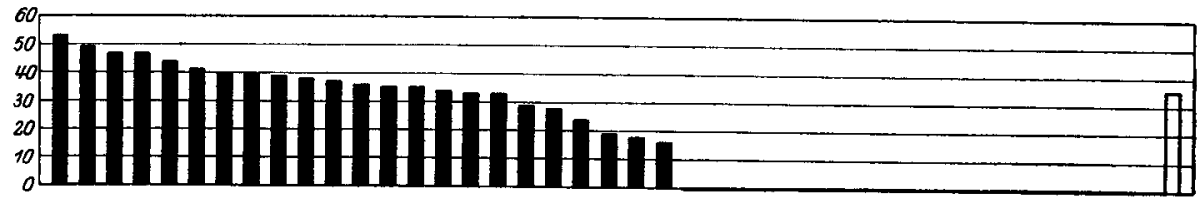

Abb. 7. Verhältnis des Globulins zum Gesamteiweiß in \%. Epilepsie.

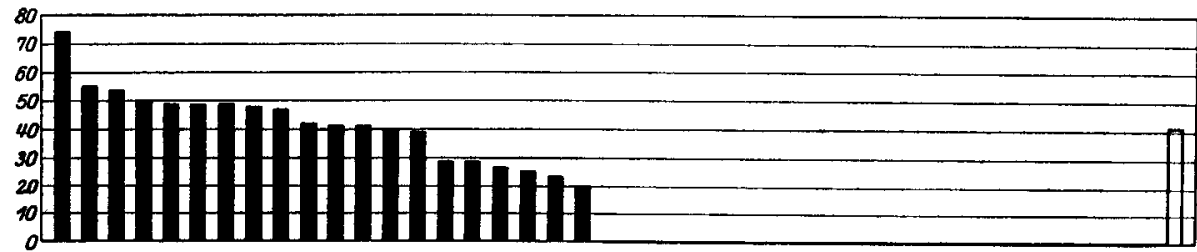

Abb. 8. Verhältnis des Globulins zum Gesamteiweib in $\%$. Paralyse.

Auch Blutgerinnungszeit und antitryptischer Serumtiter (Tafeln 9 bis 12 und 13-16) zeigen gegenüber den anderen Psychosen keine einheitliche Abweichung und wir können deshalb den aus den Resultaten dieser beiden Reaktionen von anderer Seite gezogenen Schlußfolgerungen nicht beistimmen.

Zur Morphologie des Blutes. Die Tafeln 17-36 zeigen die Zahlen für rote Blutkörperchen, Hämoglobin und Leukocyten, sowie die relativen Zahlen für Lymphocyten und eosinophilen Zellen. Irgendwelche wesentliche Abweichungen haben sich bei diesen Untersuchungen nicht ergeben. Die Schultzsche capilläre Erythrostase können wir ebensowenig bestätigen, wie wir den aus dem Verhalten der Leukocyten, Lymphocyten und eosinophilen Zellen gezogenen Schlüssen auf bestimmte Konstitutionsanomalien oder Toxinwirkungen beitreten können. 
O. Wuth:

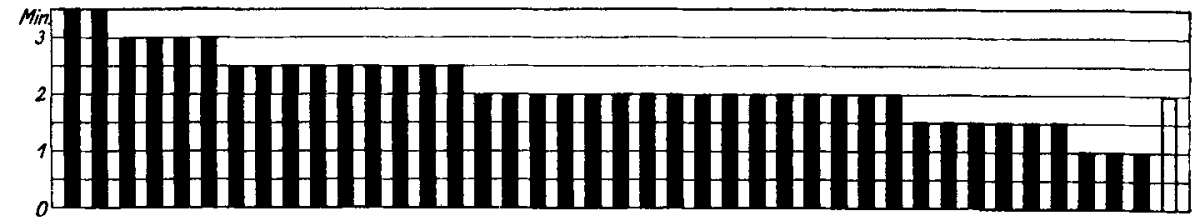

Abb. 9. Gerinnungszeit. Melancholie.

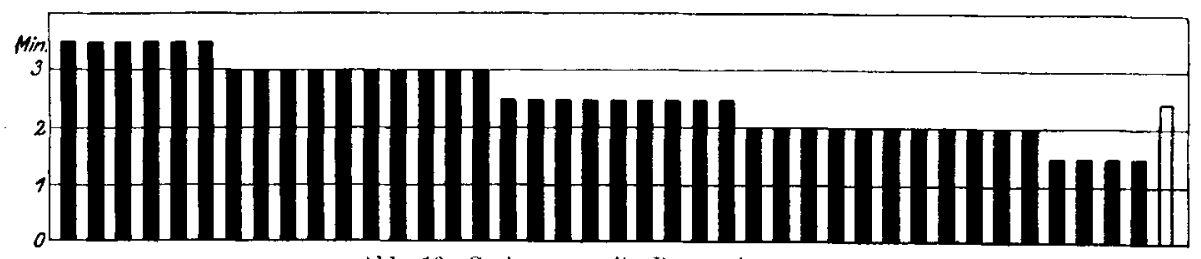

Abb. 10. Gerinnungszeit. Dementia praecox.

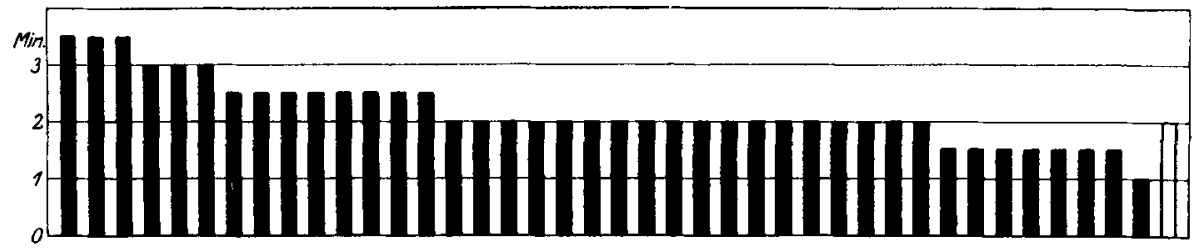

Abb. 11. Gerinnungszeit. Epilepsie.

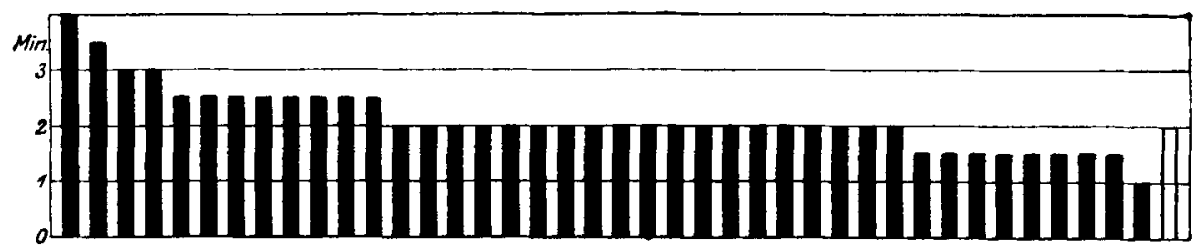

Abb. 12. Gerinnungszeit. Paralyse.

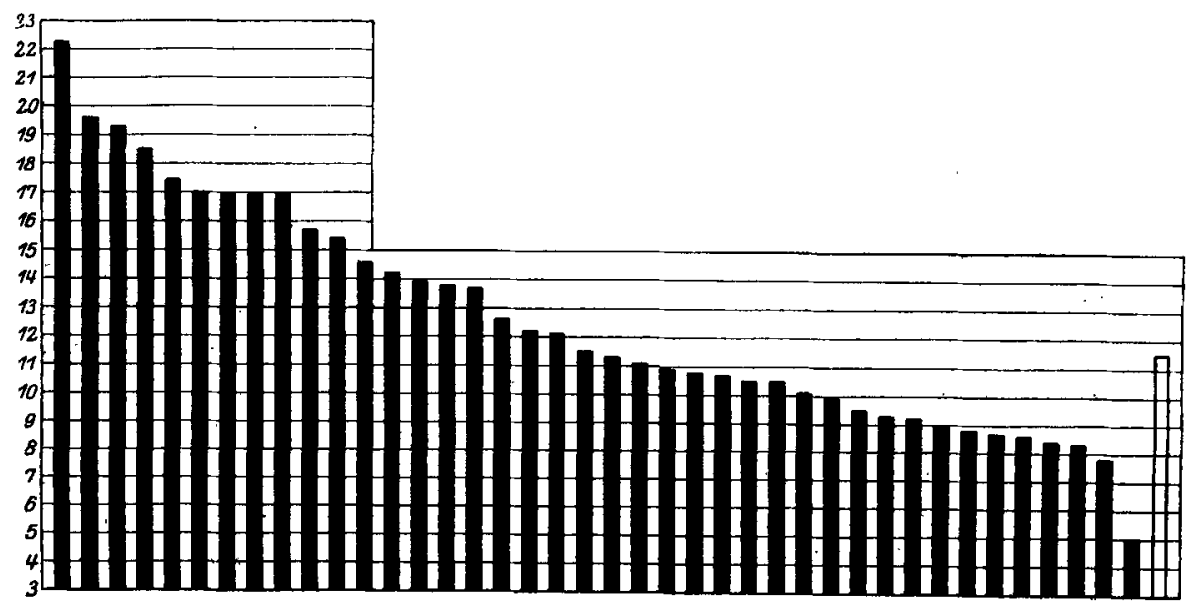

Abb. 13. Antitryptischer Index. Melancholie. 


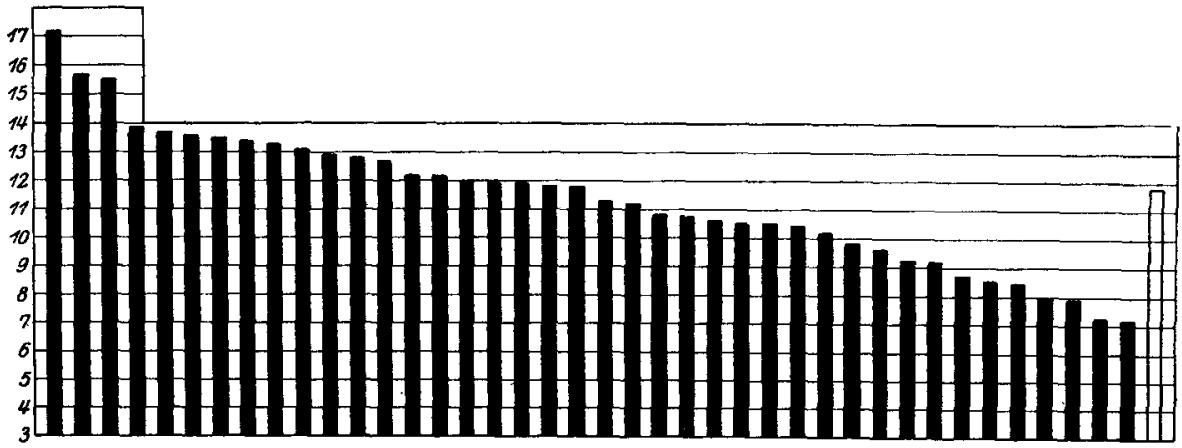

Abb. 14. Antitryptischer Index. Dementia praecox.

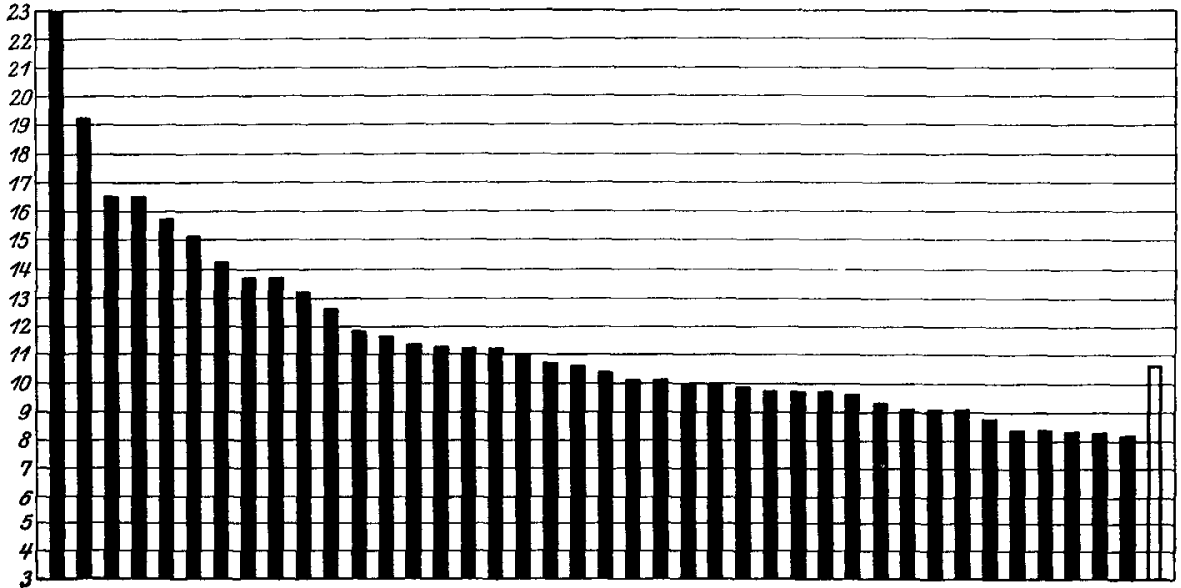

Abb. 15. Antitryptischer Index. Epilepsie.

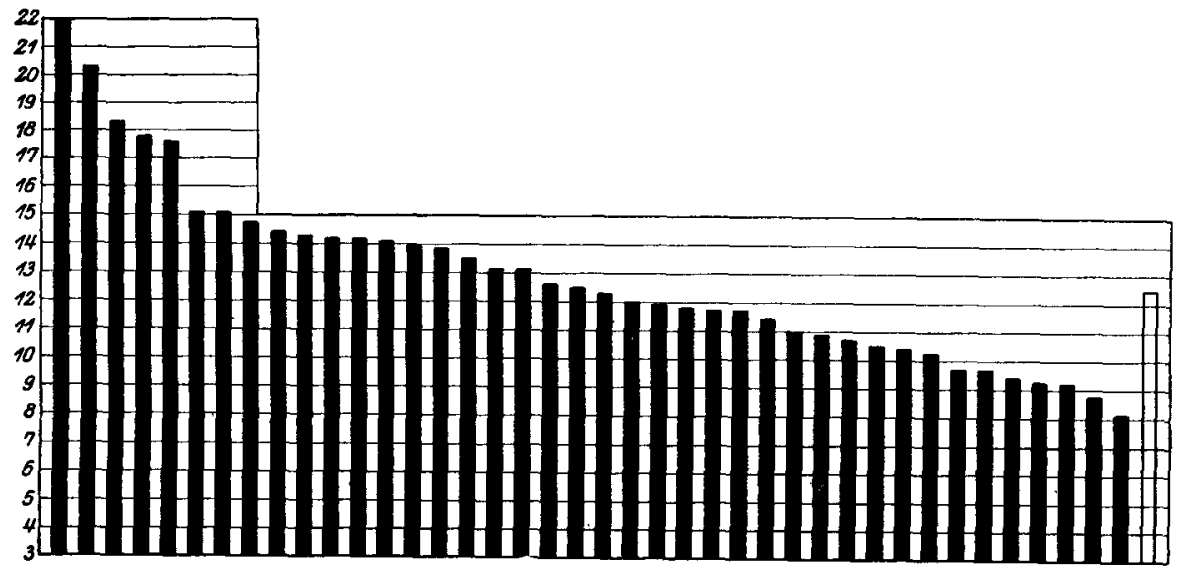

Abb. 16. Antitryptischer Index. Paralyse. 


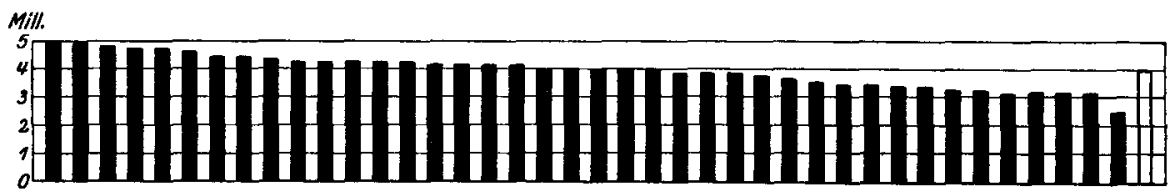

Abb. 17. Erythrocyten. Melancholie.

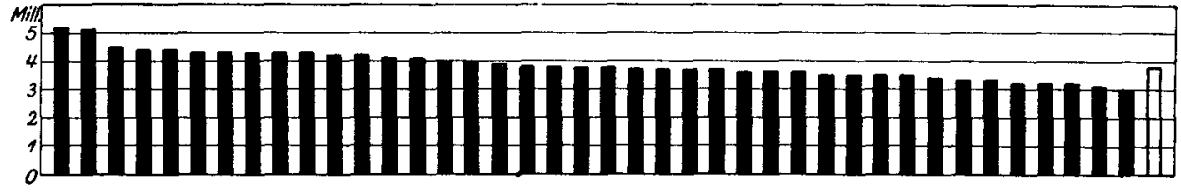

Abb. 18. Erythrocyten. Dementia praecox.

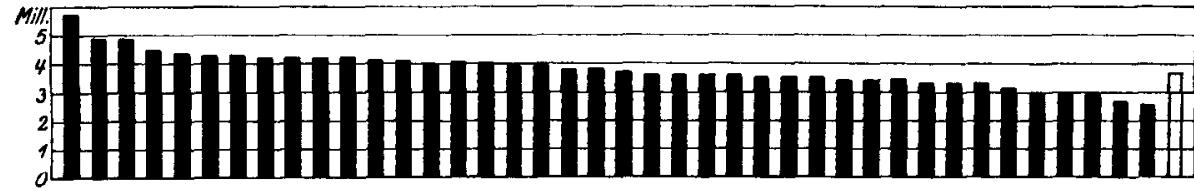

Abb. 19. Erythrocyten. Epilepsie.

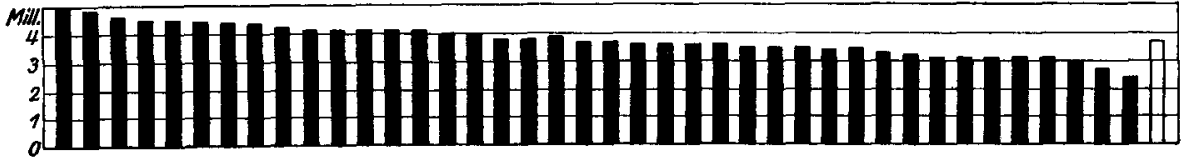

Abb. 20. Erythrocyten. Paralyse.

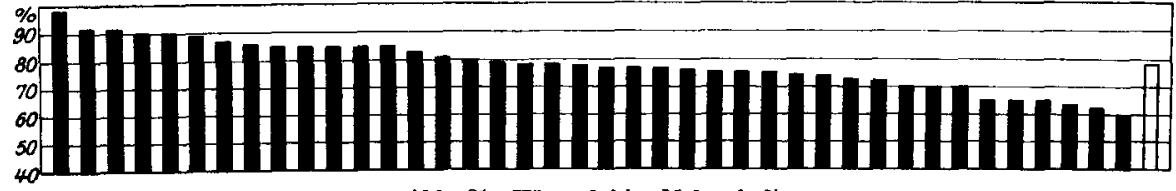

Abb. 21. Hämoglobin. Melancholie.

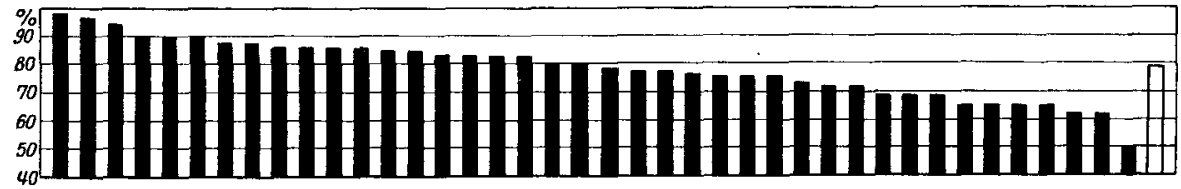

Abb. 22. Hämoglobin. Dementia praecox.

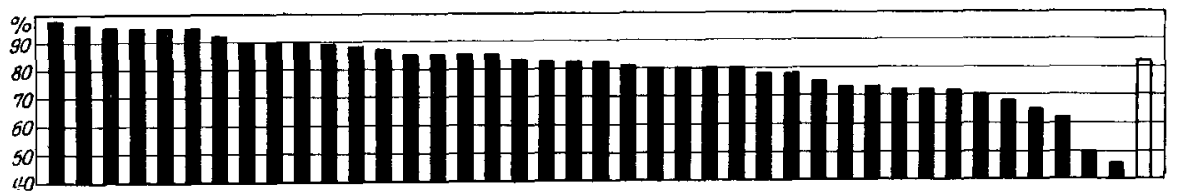

Abb. 23. Hämoglobin. Epilepsie. 


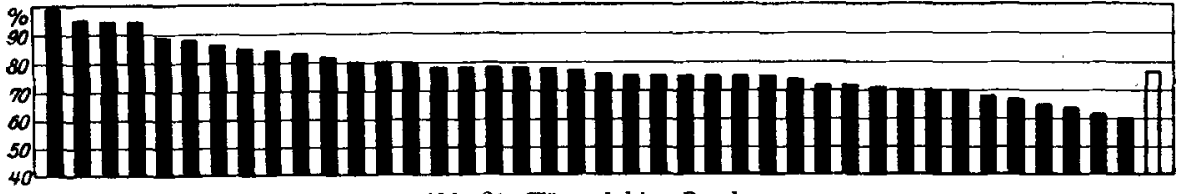

Abb. 24. Hämoglobin. Paralyse.

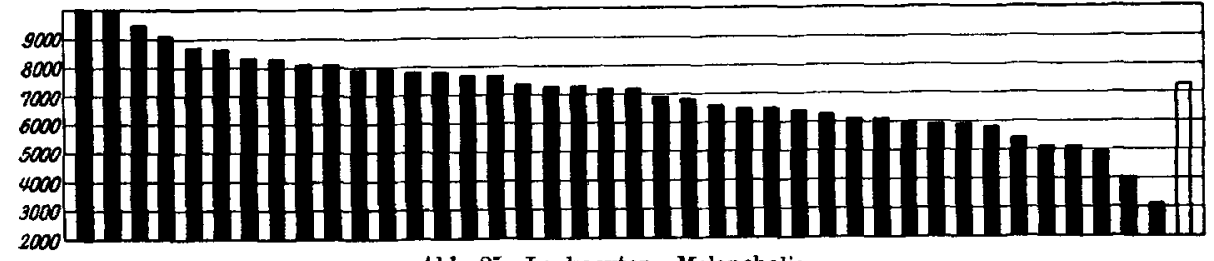

Abb. 25. Leukocyten. Melancholie.

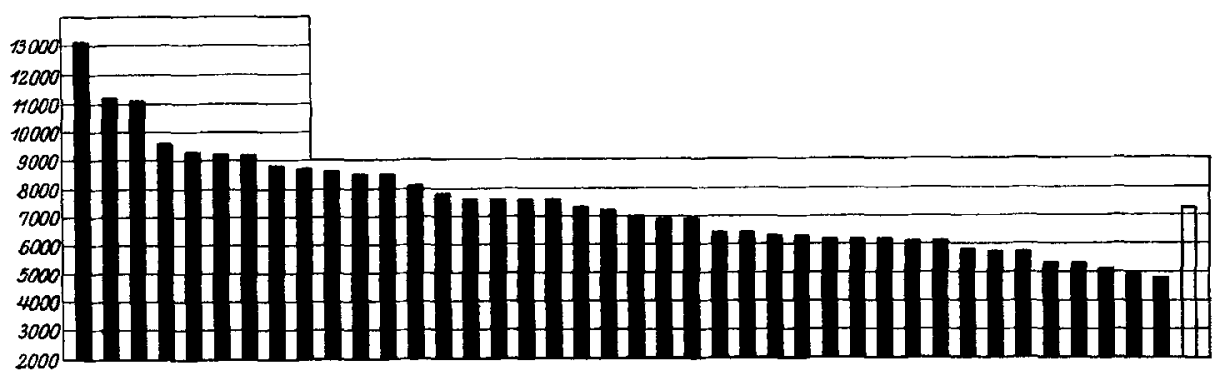

Abb. 26. Leukocyten. Dementia praecox.

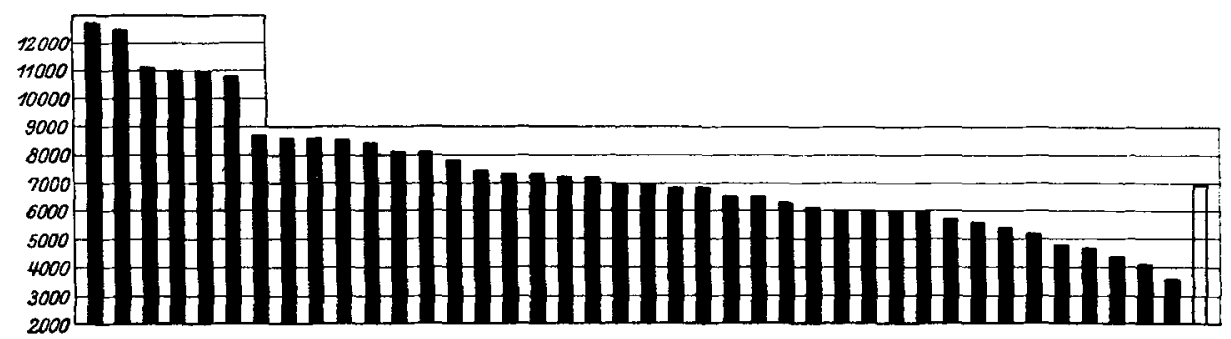

Abb. 27. Leukncyten. Epilepsie.

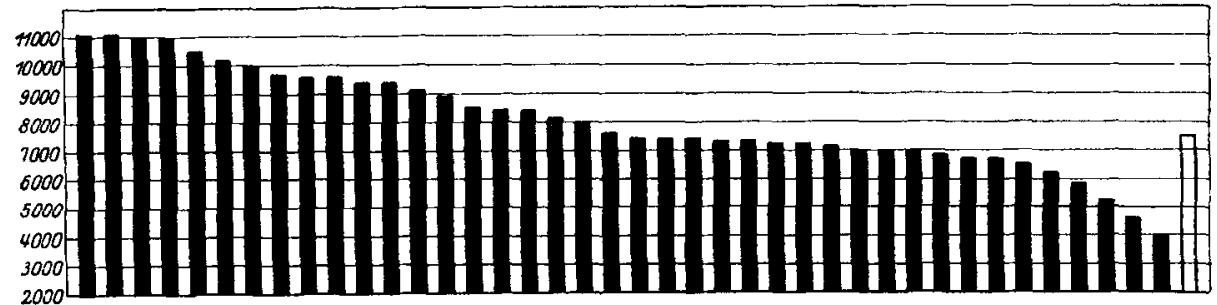

Abb. 28. Leukocyten. Paralyse. 


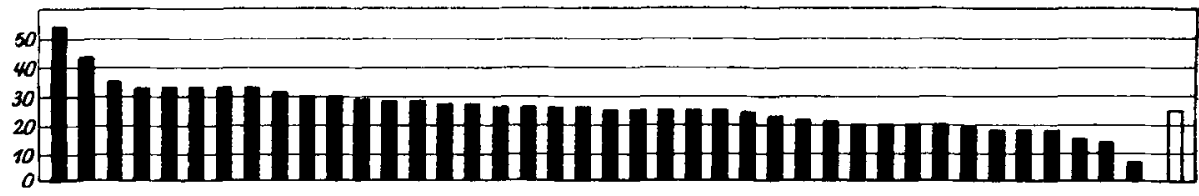

Abb. 30. Lymphocyten. Dementia praecox.

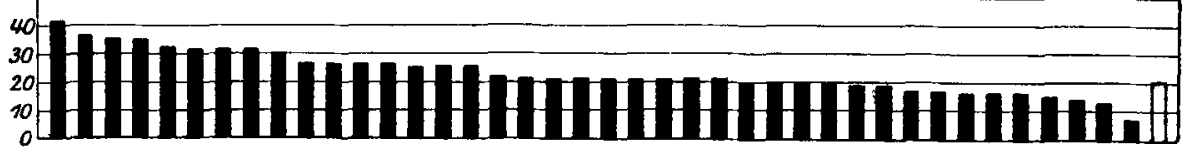

Abb. 81. Lymphocyten. Epilepsie.

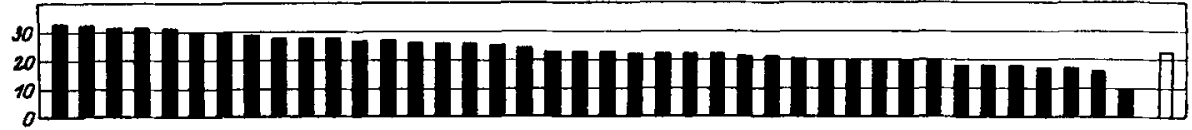

Abb. B2. Lymphocyten. Paralyse.

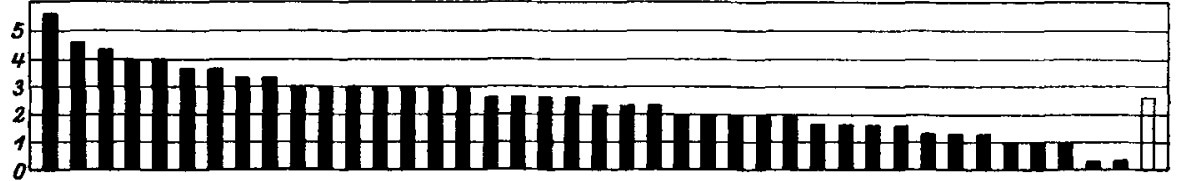

Abb. 39. Eosinophile. Melancholie.

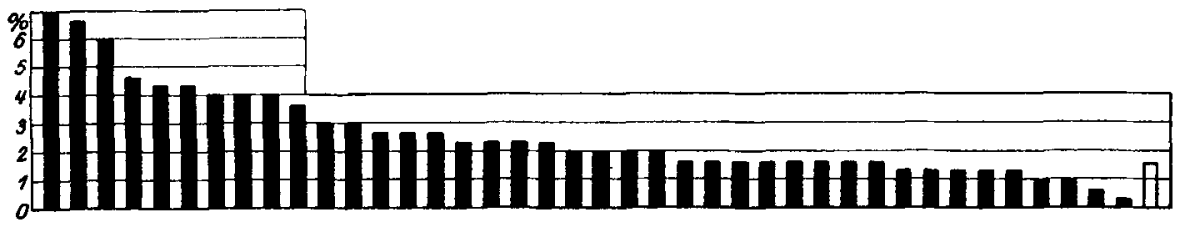

Abb. 34. Eosinophile. Dementia praecox.

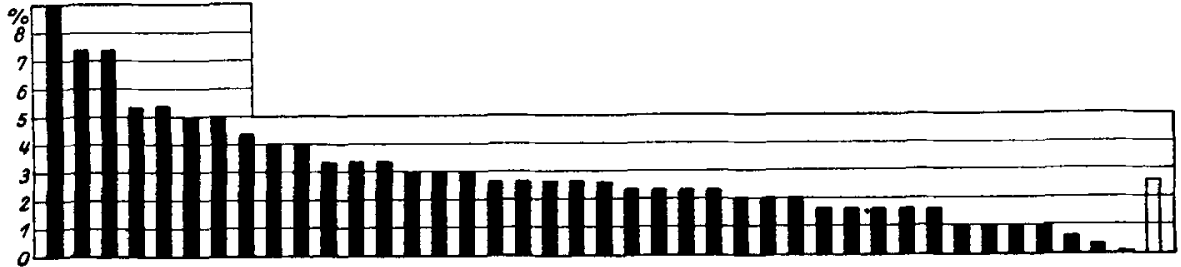

Abb. 35. Eosinophile. Epilepsie. 


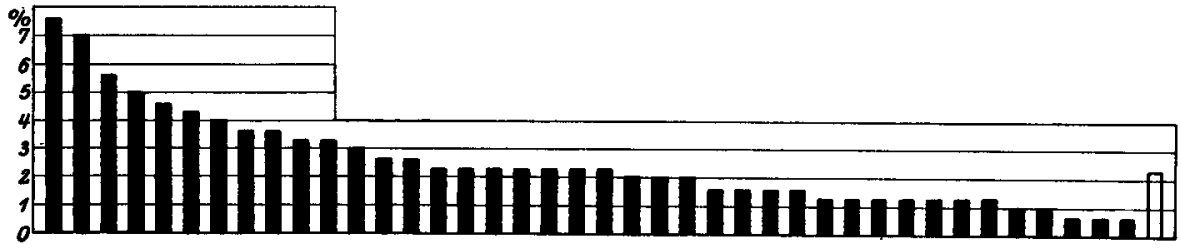

Abb. 36. Eosinophile. Paralyse.

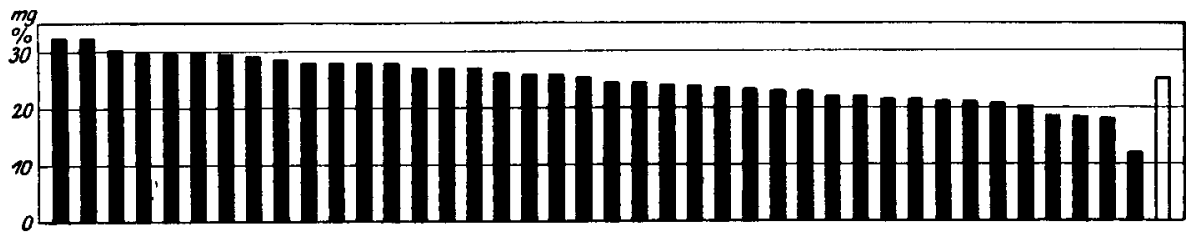

Abb. 37. Reststickstoff. Melancholie.

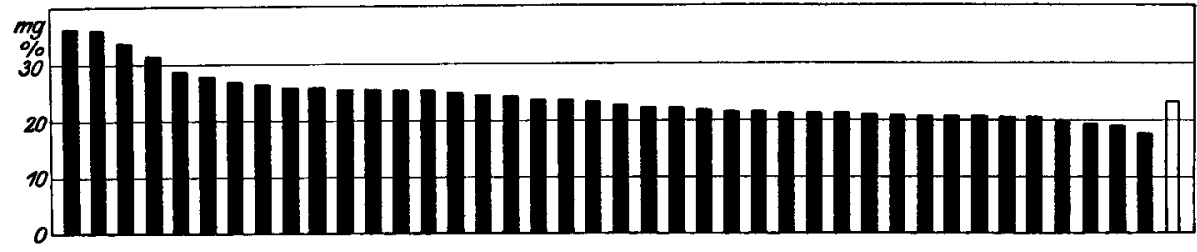

Abb. 38. Reststickstoff. Dementia praecox.

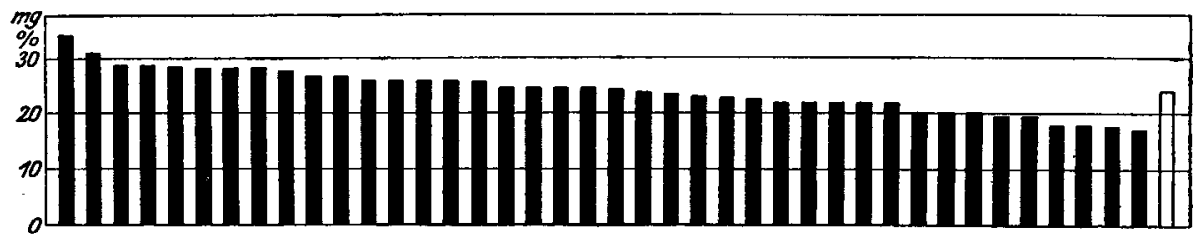

Abb. 3^. Reststickstoff. Epilepsie.

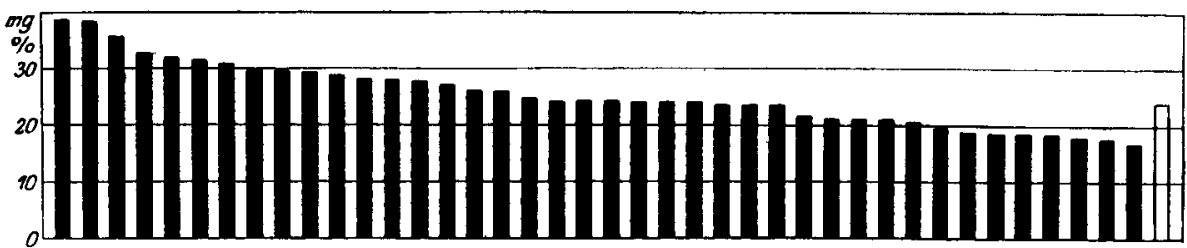

Abb. 40. Reststickstoff. Paralyse.

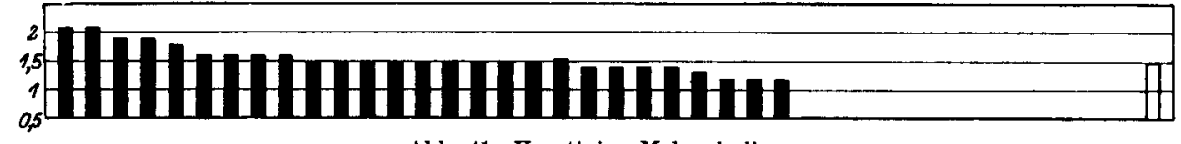

Abb. 41. Kreatinin. Melancholie. 
o. Wuth:

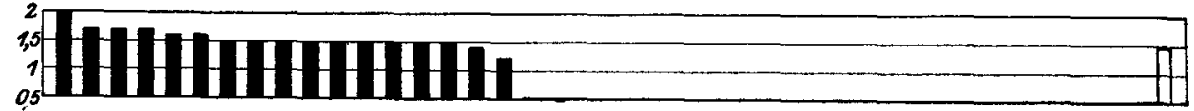

Abb. 42. Kreatinin. Dementia praecox.

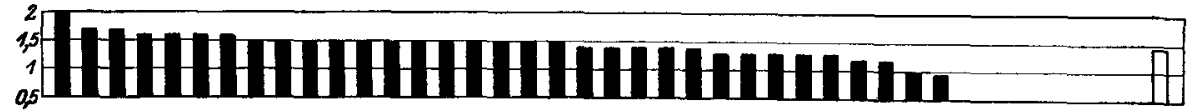

Abb. 43. Kreatinin. Epilepsie.

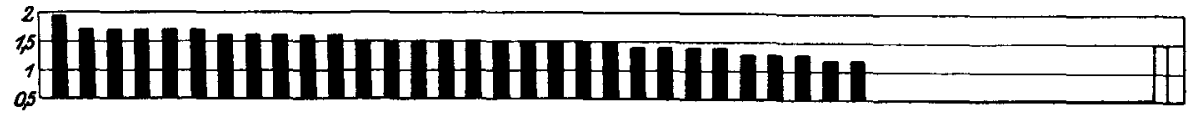

Abb. 44. Kreatinin. Paralyse.

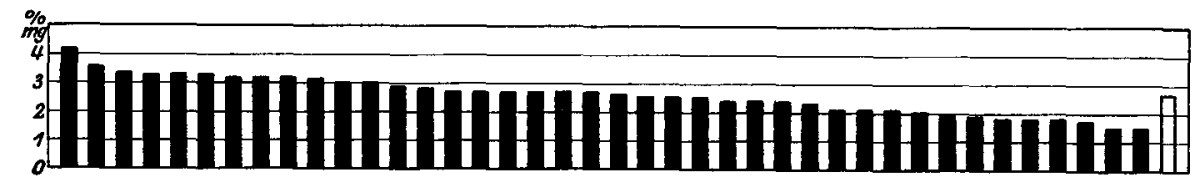

Abb. 45. Harnsäure. Melancholie.

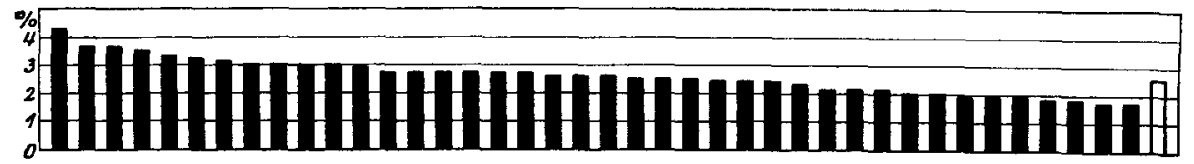

Abb. 46. Harnsäure. Dementia praecox.

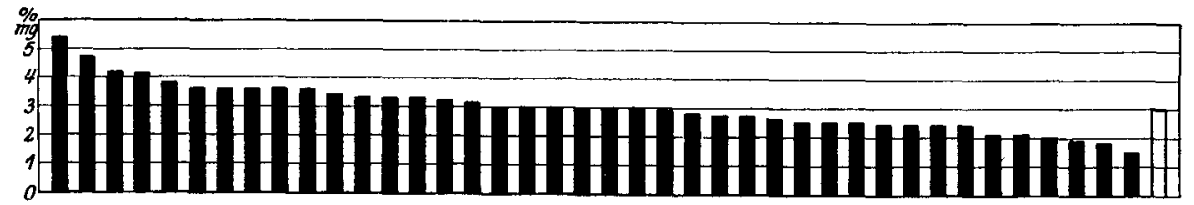

Abb. 47. Harnsăure. Epilepsie.

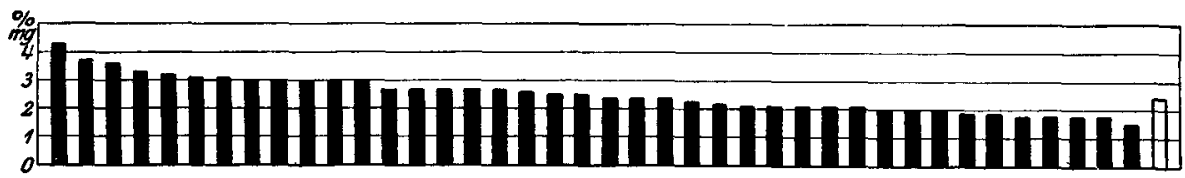

Abb. 48. Harnsäure. Paralyse. 
Untersuchungen über die körperlichen Störungen bei der Schizophrenie. 543

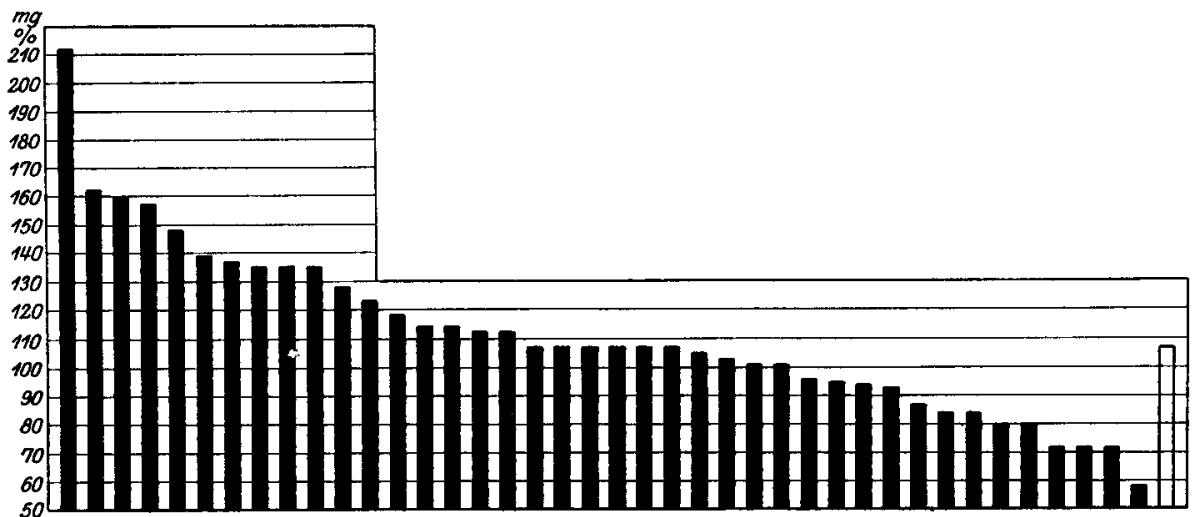

Abb. 49. Zucker. Melancholie.

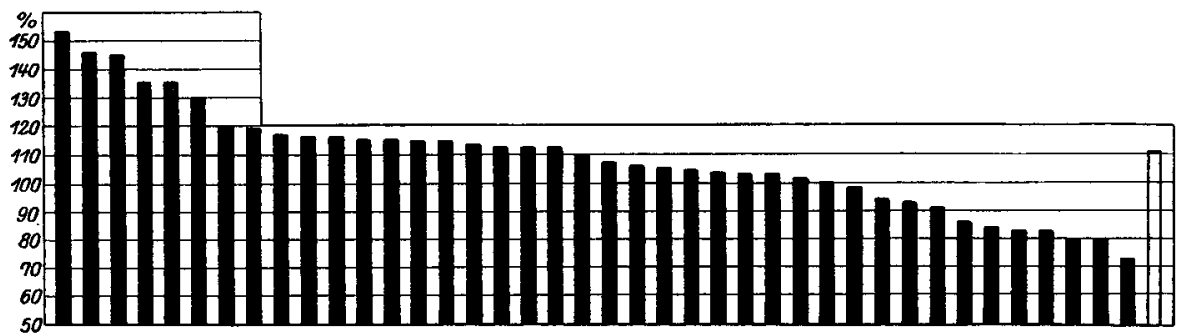

Abb. 50. Zucker. Dementia praecox.

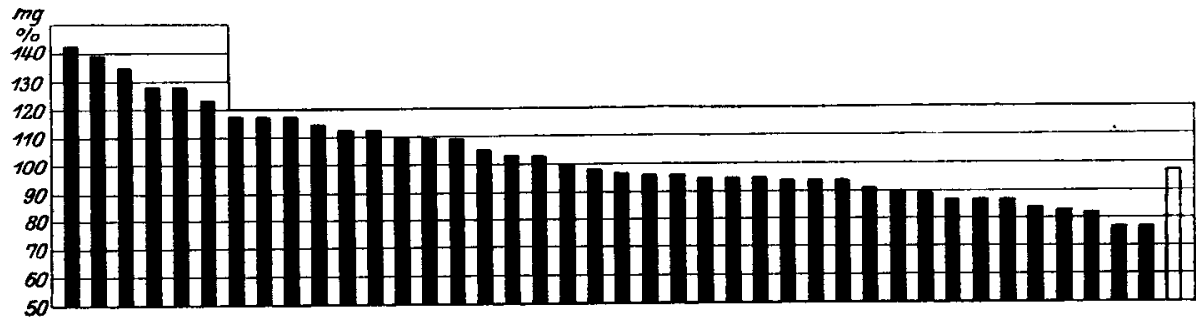

Abb. 51. Zucker. Epilepsie.

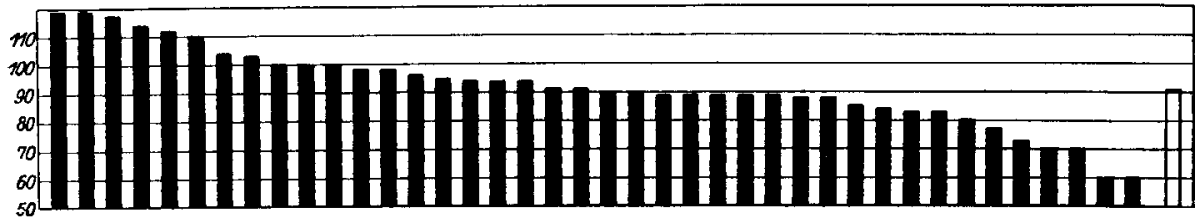

Abb. 52. Zucker. Paralyse. 
Wir kommen nun zum Chemismus des Blutes.

Auf Tafeln 37-48 sind die Werte für Rest-Stickstoff, Kreatinin und Harnsäure, und zwar in mg Prozent dargestellt. Die Werte liegen durchweg innerhalb des Rahmens der Norm und geben für die Pathogenese der Schizophrenie keinerlei Anhaltspunkte, sei es für gesteigerten Zerfall, sei es für eine Retention auf dem Gebiete des Eiweiß- oder Purin-Stoffwechsels. Damit entfallen die Grundlagen der Hypothesen, welche eine Retention für die Entstehung der Dementia praecox verantwortlich machen möchten.

Anders als die bisher erwähnten Bestandteile verhält sich der Blutzucker.

Aus den Tafeln 49-52 ist ersichtlich, daß der Blutzucker Erhöhungen zeigt, welche im Durchschnitt die bei der Melancholie gefundenen Werte noch etwas übertreffen, wobei daran erinnert sei, daß namentlich von englischen Autoren die Hyperglykämie als Affekthyperglykämie bis zu einem gewissen Grade der Melancholie eigen sein soll. Nun müssen wir unseren heutigen Kenntnissen nach für die meisten Formen von Hyperglykämie eine Mitwirkung des endokrinen Systems annehmen. Und so kann vielleicht diese Abweichung von der Norm als eine gewisse Stütze der Hypothese erscheinen, die uns z. Z. mangels anderer Erklärungsmöglichkeiten als die plausibelste vorkommt, nämlich die der endokrinen Genese der Dementia praecox.

Ebenfalls in diesem Sinne zu verwerten sind vielleicht die Gewichtsschwankungen, namentlich im Sinne der endogenen Mast, die Wasserschwankungen und vielleicht die von Bornstein und Grafe beobachtete Herabsetzung des Energiestoffwechsels. In ähnliche Richtung scheinen ferner die Resultate der Abderhaldenschen Reaktion zu weisen, ohne daß jedoch hier die Frage der praktischen Verwertbarkeit dieser Reaktion angeschnitten sei. Und schließlich ist die Hypothese von der endokrinen Genese der Schizophrenie die einzige, welche die von der Vererbungslehre ermittelten Tatsachen, sowie das relativ häufigere Vorkommen von Dysharmonien des körperlichen Habitus bei der Dementia praecox einigermaßen zu erklären vermag.

Daß zwischen Konstitution und endokrinem System enge Beziehungen bestehen, habe ich an anderer Stelle nachzuweisen versucht und in diesem Sinne möchte ich auch nach dem heutigen Stande unserer Kenntnisse eine Beteiligung des endokrinen Systems am Zustandekommen des Krankheitsprozesses der Schizophrenie annehmen.

Haben also auch meine oben ausgeführten Untersuchungen dem erstrebten differential-diagnostischen Ziele gegenüber im wesentlichen ein negatives Resultat gezeitigt, so kann man doch vielleicht von ihnen sagen, daß sie eine klarere Grundlage für weitere Untersuchungen geschaffen haben. Ich darf sagen, daß vielleicht ihr Hauptwert darin 
liegt, vor Augen zu führen, daß unsere Kenntnisse in somato-pathologischer Hinsicht heute noch die allerdürftigsten sind, und auch für ein weiteres Eindringen nur ganz geringe Anknüpfungspunkte geben. Unserer Überzeugung nach müssen wir, wenn wir ehrlich sind, zugeben, daß wir über die somatische Pathologie der Schizophrenie heute überhaupt nichts Positives wissen und auch für die Hypothese einer endokrinen Genese keine Beweise, sondern nur einige Anhaltspunkte erbringen können. Bei diesem Stand unserer Kenntnisse will es mir für die Zukunft am aussichtsreichsten erscheinen, mit biologischen und stoffwechselchemischen Methoden nach konstitutionell-endokrinen Abweichungen zu suchen, die möglicherweise zu einer haltbareren Aufstellung gewisser körperlicher Konstitutionstypen führen könnten, als die mehr gefühlsmäßig das Richtige ahnende, als auf exakten Methoden beruhende, rein äußerlich-morphologische Betrachtung es bislang vermocht hat. 